gen sei, wenn mit den Mitteln des Strafrechts $(\$ 2$ 1 $8 \mathrm{StGB})$ und Rückwärtsentwicklungen des Familienrechts Frauen und in gewisser Weise auch Männer von ihrer Vertragsautonomie auf traditionelle Geschlechtsrollen gedrängt werden, dann haben wir damit Belege für die fortbestehende Virulenz dieser Position. Sie zeigt sich immer, wenn vermeintliche oder reale biologische oder andere Besonderheiten (Rechts-) Politik und staatliche Organisation leiten sollen, meines Erachtens also auch, wenn etwa die (Geschlechts-)Differenz zum Maßstab für juristische Argumentation bis hin zu einem Verfassungsprinzip erhoben werden soll.

Ich habe sie reaktionär genannt. Das deshalb, weil sie mit rückwärtsgewandten und gleichzeitig strukturnotwendig vergeblichen Mitteln gegen » Kapitalismus oder Industriegesellschaft « und deren Freiheiten und Zwänge, Emanzipationen und Isolationen anrennen. Wo sie mit frustrierten Massen die politische Oberhand gewinnen, können sie für eine Weile ihre Konzepte ausprobieren, was wegen des unvermeidbaren Scheiterns immer zu Repression, Terror und Schrecken gegenüber durch Konkretheitsideologie Ausgegrenzten führen muß. Hoffentlich stehen wir nicht wieder am Beginn eines neuen Großversuchs in dieser Richtung. Es lohnt sich, das strockene und blutleeres 2. Buch des BGB jedenfalls gegen diese Position zu verteidigen.

Rolf Knieper

\title{
Strafrechtliche Reaktionen auf rechtsradikale Gewalttaten
}

I. Strafrechtlich ein reaktives Recht. Wann immer die Dinge sich so entwickeln, daß ihre Schädlichkeit offenkundig wird, wird in der Öffentlichkeit nach dem Strafrecht gerufen. Erst wenn Kriegswaffen ihrem bestimmungsgemäßen Gebrauch zugeführt werden, pflegt aufzufallen, wie sozialschädlich, oder besser -vernichtend ihre Wirkung ist. Daraus wird allgemein nicht der naheliegende Schluß gezogen, nun die Produktion solcher Waffen einzustellen oder ihren Einsatz unmöglich zu machen. Vielmehr wird die Strafdrohung für unerlaubte Ausfuhr geringfügig erhöht. So geschah es nach dem Golfkrieg. Wir bauen in Kenntnis der Risiken Kernkraftwerke, wiegen uns aber in Sicherheit, weil es Gesetze gibt, die die unerlaubte Herbeiführung einer Kernexplosion mit Strafe bedrohen. Wir zerstören das ökologische Gleichgewicht unserer Welt und rufen nach schärferen Strafen für Umweltsünder, wenn wir erkennen, daß wir daran zugrundegehen können. Die Liste läßt sich beliebig verlängern.

Ein besonders groteskes Beispiel für diesen irrationalen Glauben an die Herstellung persönlicher und gesellschaftlicher Sicherheit haben unsere Politiker geboten, als sie unausweichlich und öffentlich mit den Folgen rechtsradikalen Terrors konfrontiert wurden. Sie haben auf die unzureichenden strafrechtlichen Regelungen verwiesen und Verschärfungen in Aussicht gestellt, um diese Auswüchse verhindern zu können. Daran ist nicht viel Richtiges.

1. Der Ausbruch rechtsradikaler Gewalt ist, ebensowenig wie das Sterben unserer Wälder, eine unerwartete Naturkatastrophe, sondern Kulmination einer Entwicklung, die sich seit längerem abzeichnet und vor der die Öffentlichkeit die Augen verschlossen hat, selbst dann noch, als es das erste Todesopfer gab, den Libanesen Amadeu. Nur zwei Bundesländer, Hamburg und Nordrhein-Westfalen, haben ihre legalen Ausforschungsmittel, die Ämter für Verfassungsschutz, angewiesen, rechte Gruppierungen einschließlich der Republikaner auszuforschen, weil sie - mit den Worten des nordrhein-westfälischen Innenministers - davon ausgingen, daß hier unter der Maske des Biedermannes geistige Brandstiftung betrieben wird. 
2. In ihrem Eifer, mit dem Politiker nach Strafgesetzen gerufen haben, haben sie völlig übersehen, daß bereits das bestehende Strafrecht eine komplette und nahezu flächendeckende Verfolgung der Urheber und der Claqueure gewalttätiger, gegen Menschen gerichteter Aktionen ermöglicht, wie wir sie tagtäglich im Umfeld solcher Gebäude beobachten können, in denen ausländische Menschen wohnen. Wir haben nahezu mehr an Strafnormen, als einer freiheitlichen Gesellschaft guttut.

3. Der Ruf nach neuen Strafvorschriften hat also einmal mehr deutlich gemacht, daß Strafgesetze als eine Art Blitzableiter für politisches und gesellschaftliches Versagen benutzt werden.

Um es kurz zu machen: Das Strafrecht kann rechtsradikale Gewaltaktionen nicht verhindern. Es kann erst Recht die Ursachen nicht beseitigen, die den Nährboden dieser Entwicklung bilden. Wer also meint, mit Strafgesetzen die Ursachen rechtsradikaler und rassistischer Gewaltausbrüche in den Griff zu bekommen, verhält sich ähnlich irrational wie ein Analphabet, der glaubt, er könne sein Problem mit einer Lesebrille beheben.

II. Mit meiner Einführung habe ich nun lediglich vor dem Glauben an die Wirksamkeit und Heilkraft des Strafrechts warnen wollen. Ich würde gründlich mißverstanden, wenn daraus der Schluß gezogen würde, daß das Strafrecht gegen rechtsradikale Gewalttäter nicht angewendet werden sollte. Ich bin weit davon entfernt, die Lösung des Problems in dem Einsatz eines Heeres von Sozialarbeitern zu sehen, die das Gespräch mit den verirrten jungen Menschen suchen sollen, wie es einige Politiker nach den Ereignissen in Rostock vorgeschlagen haben. Vielmehr halte ich es für illegal, wenn die Mittel des Strafrechts nicht eingesetzt würden, denn die bestehenden Strafnormen sind geltendes Recht, deren Anwendung sich nach den Regeln des Strafprozeßrechts richtet.

r. Wir alle kennen den Satz, daß es keinen Anspruch auf Gleichbehandlung im Unrecht gibt. Wohl aber gebietet Art. 3 GG die Gleichbehandlung des Unrechts. Dieser spezielle Gehalt des Art. 3 GG gehört zu den tragenden Strukturprinzipien unseres Strafprozesses. Diese These ist erläuterungsbedürftig:

Strafe setzt nach den grundgesetzlichen Verfahrensgarantien einen richterlichen Urteilsspruch voraus. Da wir einen Anklageprozeß haben, darf ein Gericht sich mit einer Strafsache aber erst befassen, wenn Anklage erhoben wird. Das Anklagemonopol hat von Gesetzes wegen die Staatsanwaltschaft. - Weil schon dem historischen Gesetzgeber die Gefahren eines Monopols geläufig waren, hat er diese Gefahren durch das sogenannte Legalitätsprinzip einzudämmen gesucht. Das Legalitätsprinzip verpflichtet die Staatsanwaltschaft und die für sie als »verlängerter Arm《 tätige Polizei, bei Vorliegen von Anhaltspunkten für eine strafbare Handlung ausnahmslos und ohne Ansehen von Person und Stand die Ermittlungen aufzunehmen. Es verpflichtet die Staatsanwaltschaft ferner, Anklage zu erheben, wenn die Ermittlungen einen hinreichenden Tatverdacht ergeben. Dieser sogenannte "Verfolgungszwang" gilt uneingeschränkt. Lediglich im Bereich der Bagatelldelikte und der kleineren bis mittleren Kriminalität ist es der Staatsanwaltschaft gestattet, das Verfahren einzustellen, also von der Anklage abzusehen, nicht aber schon von der Aufnahme der Ermittlungen.

2. Ich meine nun, daß diese Pflicht zur Gleichbehandlung des Unrechts nicht ernst genommen wird, wenn es um die Verfolgung rechtsradikaler Gewalttaten geht.

Die Pflicht entsteht bei Verdacht einer Straftat. Ein solcher Verdacht, und damit komme ich auf die Strafgesetze zurück, drängt sich bei der Beobachtung des schon fast klassischen Ablaufs speziell gegen Ausländer gerichteter Gewaltaktionen geradezu auf. 
- Mord, $\iiint_{211,212,22} S t G B$ : Wer einen Brandsatz in ein von Menschen bewohntes Gebaude wirft, erfüllt, wenn durch glückliche Fügung kein Mensch dabei zu Tode kommt, jedenfalls alle Voraussetzungen des versuchten Mordes. JuristInnen wissen, daß es für vorsätzliches Verhalten und mithin für die subjektiven Versuchsvoraussetzungen ausreicht, wenn nur mit der Möglichkeit des tatbestandlichen Erfolges - bei Mord also mit der Möglichkeit des Todes anderer Menschen - gerechnet und diese Möglichkeit billigend in Kauf genommen wird. Daß ein solches Verhalten darüber hinaus alle Merkmale aufweist, die die (versuchte) Tötung als Mord qualifizieren, dürfte offensichtlich sein. Brandsätze in die Zimmer schlafender Menschen zu werfen, ist »heimtückisches « Verhalten; Brandsätze sind als Tötungsmittel darüber hinaus "gemeingefährlich «; und schließlich dürften sich auch die Motive für ein solches Verhalten als »niedrige Beweggründe«, d. h. nach allgemeiner Auffassung auf sittlich niedrig ster Stufe stehende Gründe für die Tat, beurteilen lassen.

- Schwere und besonders schwere Brandstiftung, $\iint$ 306, 307 StGB: Das Inbrandsetzen eines Gebäudes, das zur Wohnung von Menschen dient, ist ein Verbrechen, auch wenn dabei niemand zu Schaden kommt. Deshalb ist auch schon der Versuch einer solchen Brandstiftung strafbar. Kommt dabei ein Mensch zu Tode ist die Freiheitsstrafe gar lebenslänglich.

- Volksverhetzung, $\int$ Izo StGB: Mit mindestens 3 Monaten Freiheitsstrafe ist zu bestrafen, wer in einer Weise, die geeignet ist, den öffentlichen Frieden zu stören, die Menschenwürde durch folgende Maßnahmen angreift: entweder "Aufstacheln zum Haß gegen Teile der Bevölkerung o oder "Aufforderung zu Gewalt- oder Willkürmaßnahmen gegen Teile der Bevölkerung « oder »Beschimpfen, böswilliges Verächtlichmachen oder Verleumden« von Teilen der Bevölkerung. Die schlichte These, alle Ausländer seien »Asylanten « reicht danach, denn dieser Begriff suggeriert, daß es sich ausschließlich um Personen handelt, die unter Mißbrauch des deutschen Asylrechts wirtschaftliche Vorteile erstreben. Man braucht nicht erst an die Flüchtlinge aus den Kriegsgebieten des Balkans zu denken, um zu erkennen, daß darin eine Verleumdung liegt.

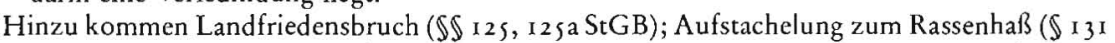
StGB), Bildung krimineller oder terroristischer Vereinigungen $(\$ \mathbf{1} 29$, I 29a StGB); die Liste möglicher Straftatbestande läßt sich beliebig erweitern, wenn man nur an die allgemeinen Körperverletzungstatbestände denke.

Da im deutschen Strafrecht des Territorialitätsprinzip gilt, unterliegt jede Straftat, die im Geltungsbereich dieses Gesetzes begangen wird, der deutschen Strafgewalt. Die Nationalität oder Staatsangehörigkeit des Opfers ist danach gerade kein Kriterium, das eine Einschränkung der Strafverfolgung zulassen könnte.

3. Der eigentliche Skandal liegt daher aus der Sicht des Strafrechts bei der Frage, warum sich die zur Verfolgung verpflichteten Behörden, insbesondere die Polizei, so bemerkenswerte Zurückhaltung auferlegt und über eine lange Zeit die Augen verschlossen hat. Diese Frage haben auch schon andere gestellt. So hat der Spiegel im 45. Heft ein Interview mit dem Generalbundesanwalt abgedruckt, in dem dieser u. a. dazu Stellung genommen hat, warum die rechtlichen Instrumentarien nicht eingesetzt werden, die der Gesetzgeber in den Jahren 1974 und 1976 zur Bekämpfung des (Links-) Terrorismus eingeführt hat und die bis heute geltendes Recht sind. Stahl meint, die rechtsradikale Szene könne z. B. nicht unter dem Gesichtspunkt der terroristischen Vereinigung verfolgt werden, weil es sich nicht um auf Dauer angelegte Organisationen handele, sondern um »spontane, unorganisierte Gewaltaktionen, begangen von Radikalen und Extremisten «.

Diese Aussage zeigt, daß die Annahme von »Tatverdacht« als Grundlage einer strafrechtlichen Verfolgung bereits ein Subsumtionsvorgang ist, in den alltagstheoretische (Vor-)Urteile eingehen, hier also: "Rechtsradikale sind zu einer ordnungsgemäßen Organisation nicht fähige Dummköpfe.«Solche Vorstellungen lassen sich einerseits nicht mit der Erkenntnis vereinbaren, daß es eine Vielzahl von neonazistisch geprägten Organisationen gibt, die schon seit Jahren, wenn nicht Jahrzehnten, bekannt sind - wenn man beispielsweise an die sog. »Wehrsportgruppen« denkt - und die ihrerseits - um das Eingangszitat zu wiederholen - »die geistigen Brandstifter « der heutigen Gewalteruptionen sind. Danach drängt sich lediglich die Frage auf, warum gerade der Generalbundesanwalt davon keine Kenntnis hat. Er hat davon möglicher- 
weise deshalb keine Kenntnis, weil die polizeilichen Datensammlungen, die ebenfalls zu Zeiten der Bekämpfung des (Links-)Terrorismus ausgebaut und mit enormen Datenmengen gefüllt wurden, nur wenig an Daten über die rechtsterroristische Szene enthalten. Das aber kann kein Grund dafür sein, Ermittlungen in dieser Richtung zu unterlassen. Daß sie viel zu spät erst einsetzen, zeigt das Beispiel der Überwachung der Republikaner durch den Verfassungsschutz: Nur zwei Bundesländer haben davon Gebrauch gemacht. Erst jetzt, wie vor wenigen Tagen den Nachrichten zu entnehmen war, soll das bundesweit ausgedehnt werden.

III. Mein Fazit lautet also: Wir haben aus strafrechtlicher Sicht keinen zusätzlichen Handlungsbedarf. Was wir brauchen, ist die Erinnerung daran, daß Polizei und Staatsanwaltschaft von Gesetzes wegen in Anwendung der geltenden Strafgesetze zu ihrer Verfolgung verpflichtet sind. Das Strafrecht hat danach kein sogenanntes »Ausländerproblem« sondern ein Inländerproblem: Die letztlich verfassungsrechtlich vorgegebene Pflicht zur unparteilichen Verfolgung von Straftaten, also zur Gleichbehandlung des Unrechts ist verletzt, wenn die Verfolgung davon abhängig gemacht wird, welchem Bereich des politischen Spektrums Gewalttaten zugeordnet werden.

Ursula Nelles

\section{Überlegungen zum Rechtsradikalismus '92 aus sozialpsychologischer Sicht}

In einer Phase globaler historischer Veränderungen sind die Deutschen, vor allem die Nachkriegsgenerationen, extrem verunsichert. Gründe dafür: die schon seit langem schwelende Strukturkrise, zunehmende Arbeitslosigkeit, Ausbildungs- und Wohnungsnotstand, allgemeine Entfremdungsprozesse, Europäische Einigung, Deutsche Vereinigung, Zerfall der Ostblockstaaten und resultierende Flüchtlingsströme, Afrika-Krise, Umweltzerstörung, AIDS, und und und. Der »Kalte Krieg « war zwar bedrohlich. Offenbar noch bedrohlicher ist es aber, keine klare, stabile Ordnung von Freund und Feind mehr zu haben. Es entsteht, nicht zuletzt auch durch manche unverhältnismäßige Dramatisierung in den Medien, zunehmend tiefe, subjektiv als existenzbedrohend erlebte, kollektive Angst.

Angst ist ein sehr unangenehmer Affekt. Sie muß angesichts der Ohnmacht, die Bedrohung unmittelbar zu beseitigen, innerpsychisch bewältigt, oder wie es psychoanalytisch heißt, abgewehrt werden. Zivilisierte, reife Formen der Angstbewältigung bestehen in besonnener Problemanalyse, vernünftigem gesellschaftlichen Diskurs und politischer Willensbildung hinsichtlich sinnvoller Lösungsmöglichkeiten. Je intensiver das subjektive Erleben von Bedrohung, desto stärker die Tendenz zum Zurückdrängen reifen seelischen Funktionierens, zur Regression, zu Vorurteilen, zu primitiven, irrationalen, panikartigen, gewalttätigen Reaktionsformen. Ob solche Regressionen entstehen, sich generalisieren, wie weit sie gehen - all das hängt zu einem gewissen Grade von der individuellen Persönlichkeit, sehr weitgehend aber auch von gesellschaftlichen und massenpsychologischen Einflüssen ab. Ein Hauptmerkmal solcher Abwehr ist jedenfalls das enorme unbewußte Potential der Menschen, etwas in andere Menschen oder in Situationen hineinzuphantasieren, die Tendenz zu Projektion und Abspaltung: Da wo die objektive Realität nicht unmittelbar und schnell beeinflußbar erscheint, wird sie eben ohne Aufschub psychisch umgestaltet. 